\title{
Pré-tratamento para redução dos parâmetros físico-químicos do lixiviado gerado em aterros sanitários
}

\author{
Pre-treatment for reducing the physico-chemical parameters of leached generation in sanitary land \\ Pretratamiento para la reducción de parámetros físico-químicos de lixiviaciones generadas em
} rellnos sanitarios

Recebido: 13/07/2021 | Revisado: 21/07/2021 | Aceito: 25/07/2021 | Publicado: 01/08/2021

\author{
Bruna Leite da Silva \\ ORCID: https://orcid.org/0000-0002-4675-5455 \\ Universidade Federal de Santa Maria, Brasil \\ E-mail: brunaleite.eng@gmail.com \\ Marcelo Lovato Brum \\ ORCID: https://orcid.org/0000-0003-3468-5346 \\ Universidade Federal de Santa Maria, Brasil \\ E-mail: marcelolvtb@gmail.com \\ Rafaela Pohlmann Velasquez \\ ORCID: https://orcid.org/0000-0001-8387-9986 \\ Universidade Federal de Santa Maria, Brasil \\ E-mail: rafaelapohlmann.velasquez@gmail.com \\ Brenda Dilkin de Lima \\ ORCID: https://orcid.org/0000-0001-8237-971X \\ Universidade Franciscana, Brasil \\ E-mail: brendadilkin.eng@gmail.com \\ Alexandre Swarowsky \\ ORCID: https://orcid.org/0000-0002-0787-2691 \\ Universidade Federal de Santa Maria, Brasil \\ E-mail: aleswar@gmail.com \\ Juliane dos Santos Pinto \\ ORCID: https://orcid.org/0000-0001-8061-4990 \\ Universidade Franciscana, Brasil \\ E-mail: julianepinto@ufn.edu.br
}

\begin{abstract}
Resumo
O objetivo deste trabalho foi avaliar o processo de filtragem em escala piloto no pré-tratamento do lixiviado de aterro sanitário através de análises físico-químicas. Foram avaliados quatro tipos de filtros; nos quais os materiais filtrantes utilizados foram areia fina, média e grossa, carvão ativado, argila, brita $n^{\circ} 0$ e brita $n^{\circ} 1$. A análise estatística foi realizada por análise de variância (ANOVA) seguida pelo teste de Tukey, quando aplicável. Os dados foram expressos como média \pm desvio padrão e os valores $\mathrm{P}<0,05$ foram considerados estatisticamente significativos. $\mathrm{O} \mathrm{pH}$ não obteve redução ou aumento significativo das análises dos filtros quando comparado com o lixiviado bruto, a condutividade elétrica obteve redução significativas no filtro 1 ( $p<0,001), 2$ e 4 com $p<0,05$ quando comparado com o lixiviado bruto. No oxigênio dissolvido ocorreram aumentos significativos no filtro 1 e 2 ( $p<0,001)$ e no filtro 4 $(\mathrm{p}<0,01)$. Conforme a redução dos parâmetros, pode-se observar que o filtro 1 reduziu aproximadamente $50 \%$ da condutividade elétrica e obteve aumento de $377,75 \%$ no oxigênio dissolvido, sendo esses os maiores valores de redução e aumento dos parâmetros. A vazão dos filtros foram todas diferentes, na qual o filtro 4 obteve maior vazão e o filtro 3 com a menor vazão. Conforme a análise dos resultados, o filtro considerado o mais viável em relação a vazão e com a análise dos parâmetros foi o filtro 1 .
\end{abstract}

Palavras-chave: Aterro sanitário; Processo de filtragem; Chorume; Resíduos sólidos.

\begin{abstract}
The objective of this work was to evaluate the pilot-scale filtration process in the pre-treatment of landfill leachate through physical-chemical analyzes. Four types of filters were evaluated; in which the filtering materials used were sand, medium and coarse sand, activated carbon, clay, gravel $\mathrm{n}^{\circ} 0$ and gravel $\mathrm{n}{ }^{\circ} 1$. Statistical analysis was performed by analysis of variance (ANOVA) followed by the Tukey test, when applicable. Data were expressed as mean \pm standard deviation and $\mathrm{P}$ values $<0.05$ were considered statistically significant. The $\mathrm{pH}$ did not show a reduction and a significant increase of the filter analyzes when compared to the crude leachate, the electrical conductivity obtained a significant reduction in filter 1 ( $\mathrm{p}<0.001$ ), 2 and 4 with $\mathrm{p}<0.05$ when compared to the crude leachate. In dissolved oxygen, there were significant increases in filter 1 and $2(p<0.001)$ and in filter $4(p<0.01)$. According to the reduction of the parameters, we can observe that the filter 1 reduced approximately $50 \%$ of the electrical conductivity and obtained an increase of $377,75 \%$ in the dissolved oxygen, being these the greater values of reduction and increase
\end{abstract}


of the parameters. The flow of the filters was all different, in which the filter 4 obtained higher flow and the filter 3 with the lower flow. According to the analysis of the results, the filter considered the most feasible in relation to the flow and with the analysis of the parameters was the filter 1.

Keywords: Landfill; Filtration process; Leachate; Solid waste.

\section{Resumen}

El objetivo de este trabajo fue evaluar el proceso de filtrado a escala piloto en el pretratamiento de lixiviados de vertedero mediante análisis fisicoquímicos. Se evaluaron cuatro tipos de filtros; en los cuales los materiales filtrantes utilizados fueron arena fina, media y gruesa, carbón activado, arcilla, grava ${ }^{\circ} 0$ y grava ${ }^{\circ} 1$. El análisis estadístico se realizó mediante análisis de varianza (ANOVA) seguido de la prueba de Tukey. Los datos se expresaron como media \pm desviación estándar y los valores de $\mathrm{P}<0,05$ se consideraron estadísticamente significativos. El pH no mostró reducción o aumento significativo en el análisis de los filtros en comparación con el lixiviado crudo, la conductividad eléctrica se redujo significativamente en el filtro 1 ( $\mathrm{p}<0.001$ ), 2 y 4 con $\mathrm{p}<0.05$ en comparación con el lixiviado crudo. En el oxígeno disuelto hubo aumentos significativos en el filtro 1 y $2(p<0,001)$ y en el filtro $4(p<0,01$. A medida que se redujeron los parámetros, se puede observar que el filtro 1 redujo aproximadamente el $50 \%$ de la conductividad eléctrica y obtuvo un aumento del $377,75 \%$ en el oxígeno disuelto, siendo estos los valores más altos de reducción y aumento de los parámetros. El flujo de los filtros fue diferente, en el que el filtro 4 tuvo el flujo más alto y el filtro 3 tuvo el flujo más bajo. Los resultados, el filtro considerado más viable en relación al caudal y con el análisis de los parámetros fue el filtro 1.

Palabras clave: Vertedero; Proceso de filtrado; Estiércol líquido; Residuos sólidos.

\section{Introdução}

A crescente demanda pelo consumo de produtos industrializados leva à contínua produção de lixo. Com o crescimento econômico do país, esse problema foi agravado, pois sabe-se que o aumento do consumo e de produção impactam diretamente a produção de resíduos sólidos urbanos - RSU (Ferri et al., 2015; Duan, et al., 2021). A problemática dos resíduos sólidos urbanos é desde a etapa da geração até a disposição final. Os impactos ambientais negativos decorrentes da disposição inadequada de resíduos sólidos podem provocar contaminação de corpos d'água, assoreamento, enchentes, proliferação de vetores acarretando em transmissão de doenças, incluindo também a poluição visual, mau cheiro e contaminação do ambiente (Mucelin; Bellini, 2008; Dhar-chowdhury et al., 2015; Souza, 2020).

Uma alternativa viável para o destino dos resíduos é o aterro sanitário de resíduos sólidos urbanos. A NBR 8419 (Associação brasileira de normas técnicas, 1992) define como técnica de disposição de resíduos sólidos urbanos no solo, sem causar danos à saúde pública, segurança e ao meio ambiente, processo que utiliza princípios de engenharia para o confinamento dos resíduos sólidos à menor área e menor volume possível, cobrindo-os com camada de terra na conclusão de cada jornada diária, ou como for necessário.

A decomposição biológica do resíduo no interior do aterro sanitário gera grande quantidade de chorume, sendo este caracterizado pela alta Demanda Bioquímica de Oxigênio $\left(\mathrm{DBO}_{5}\right)$, alto valor de Demanda Química de Oxigênio (DQO) e diversos compostos potencialmente tóxicos (Wu et al., 2017). Por possuir alto potencial de contaminação, a legislação vigente adota parâmetros a serem seguidos, necessitando de tratamentos eficientes para que o chorume possa ser lançado aos corpos hídricos ou para ser utilizado em condições não potáveis (Castilhos junior et al., 2010, Leite et al., 2021). Este lixiviado é um líquido contaminante gerado depois do processo de percolação de água no resíduo sólido, sendo um processo natural de umidade do resíduo (Wiszniowski et al., 2006; Araújo et al., 2020), resultado das bactérias nele presente, que expelem enzimas que dissolvem a matéria orgânica com formação de líquido, gerado no processo de decomposição (Serafim et al., 2003; Zhang et al., 2021).

A escolha do processo de tratamento depende das características do lixiviado do aterro sanitário, onde deve-se avaliar os aspectos legais, custos e tecnologias disponíveis para este tratamento (Chen et al., 2021). Os tratamentos podem ser físicoquímicos e biológicos, não há definido uma melhor técnica de tratamento devido as diferenças dos parâmetros físico-químicos de cada aterro sanitário, por isso na maioria das vezes as melhores eficiências ocorrem com a combinação dos dois processos 
citados anteriormente (Queiroz et al., 2011). Cabe ressaltar que as características do lixiviado dependerão das condições ambientais locais, tempo de disposição, forma de operação do aterro e as maneiras de despejos.

A filtração é uma operação que consiste em separar mecanicamente as partículas sólidas de uma suspensão líquida com o auxílio de um leito poroso, chamado de meio filtrante. O líquido obtido do processo de filtração é chamado de filtrado (Silva et al., 2014). Existem diversos filtros para pré-tratamento do lixiviado como o elaborado por Bidone, Povinelli, Cotrim (1997) feito em escala de laboratórios a partir de seis filtros percoladores para tratamento de lixiviado de aterro sanitário antigo, os que obtiveram melhores resultados foram os filtros de carvão ativado e com aparas de couro "wet blue". Ainda, sabe-se que a partir de pré-tratamento físico-químico de lixiviado de aterro sanitário com filtros de areia e coluna de carvão ativado evidenciaram resultados favoráveis para o seu objetivo, que era reduzir parâmetros físico-químicos para suceder com o tratamento biológico (Maler et al., 2014; Irazusta et al., 2020).

Os aterros sanitários, são considerados como o destino ambientalmente correto dos resíduos sólidos urbanos, contudo existe a preocupação com a geração do chorume, pois conforme aumenta a operação do aterro sanitário, aumenta a geração de lixiviado que, juntamente com as águas pluviais, podem aumentar a sua vazão (Dia et al., 2018). O tratamento deste lixiviado é complexo, sendo muitas vezes necessário passar por um ou mais processos avançados de tratamento para atingir os limites aceitáveis pelos órgãos reguladores. O pré-tratamento pode reduzir os parâmetros do lixiviado da drenagem do aterro sanitário, a logística de tratamento torna o processo mais fácil, resultando em lixiviado com melhor qualidade para os próximos processos de tratamento (Campos et al., 2013; Vasconcelos et al., 2017).

Sendo assim, o objetivo foi avaliar o processo de filtragem em escala piloto no pré-tratamento do lixiviado de aterro sanitário na cidade de Santa Maria, através de análises físico-químicas, a fim de facilitar tratamentos posteriores de lixiviado existentes nas empresas gerenciadoras de aterro sanitário, atingindo os parâmetros físico-químicos adequados perante a legislação vigente.

\section{Metodologia}

O lixiviado utilizado nos testes foi coletado das lagoas do aterro sanitário de Santa Maria, administrado pela empresa Companhia Riograndense de Valorização de Resíduos (CRVR). O lixiviado é oriundo da decomposição do resíduo, juntamente com a água da advinda da precipitação que é encaminhada através de drenagem para a estação de tratamento de lixiviado (ETL).

\subsection{Preparo Dos Materiais}

A montagem dos filtros fora em garrafas PET (politereftalato de etileno) de 3 litros, onde foram cortadas e lavadas para se suceder com as camadas de materiais filtrantes. Após, foi realizado o peneiramento da areia com a separação em fração fina, fração média fina, fração média grossa e areia grossa utilizando a série de peneiras $n^{\circ} 100(0,15 \mathrm{~mm}), \mathrm{n}^{\circ} 50(0,3 \mathrm{~mm}), \mathrm{n}^{\circ}$ $30(0,5 \mathrm{~mm})$ e $\mathrm{n}^{\circ} 16(1,2 \mathrm{~mm})$ conforme a NBR 7214 (Associação brasileira de normas técnicas, 1982). Para montar os filtros foram utilizados a areia fina, média e grossa, com suas respectivas granulometrias, 0,05 a $0,3 \mathrm{~mm}, 0,3$ a 1,2mm e 1,2 a 4,8mm. As frações, média fina e média grossa foram misturadas e caracterizadas como fração média.

Os agregados graúdos utilizados no filtro foram as britas $\mathrm{n}^{\circ} 0$ e $\mathrm{n}^{\circ} 1$, as quais passaram pelo processo de peneiramento e posteriormente lavadas em água corrente. A brita $\mathrm{n}^{\circ} 0$ possui a granulometria de 4,8 a $9,5 \mathrm{~mm}$ e a brita $\mathrm{n}^{\circ} 1$ de $9,5 \mathrm{a} 19 \mathrm{~mm}$.

Foram utilizados cinco tipos de materiais filtrantes para cada filtro, sendo a altura de cada um de $4,8 \mathrm{~cm}$ com espessura média de $24 \mathrm{~cm}$. Com isto, foram montados quatro tipos de filtros, compostos pelos materiais dispostos na Tabela $1 \mathrm{e}$ como mostra na Figura 1: 
Tabela 1. Materiais dispostos em cada um dos filtros.

\begin{tabular}{cc}
\hline Tratamento & Materiais \\
\hline Filtro 1 & areia fina, areia média, areia grossa, brita $\mathrm{n}^{\circ} 0$ e brita $\mathrm{n}^{\circ} 1$ \\
Filtro 2 & areia fina, areia grossa, carvão ativado, brita $\mathrm{n}^{\circ} 0$ e brita $\mathrm{n}^{\circ} 1$ \\
Filtro 3 & areia média, areia grossa, argila, brita $\mathrm{n}^{\circ} 0$ e brita $\mathrm{n}^{\circ} 1$ \\
Filtro 4 & areia média, argila, carvão ativado, brita $\mathrm{n}^{\circ} 0$ e brita $\mathrm{n}^{\circ} 1$ \\
\hline
\end{tabular}

Fonte: Autores (2021).

Figura 1. Os quatro protótipos de filtros realizados em bancada.

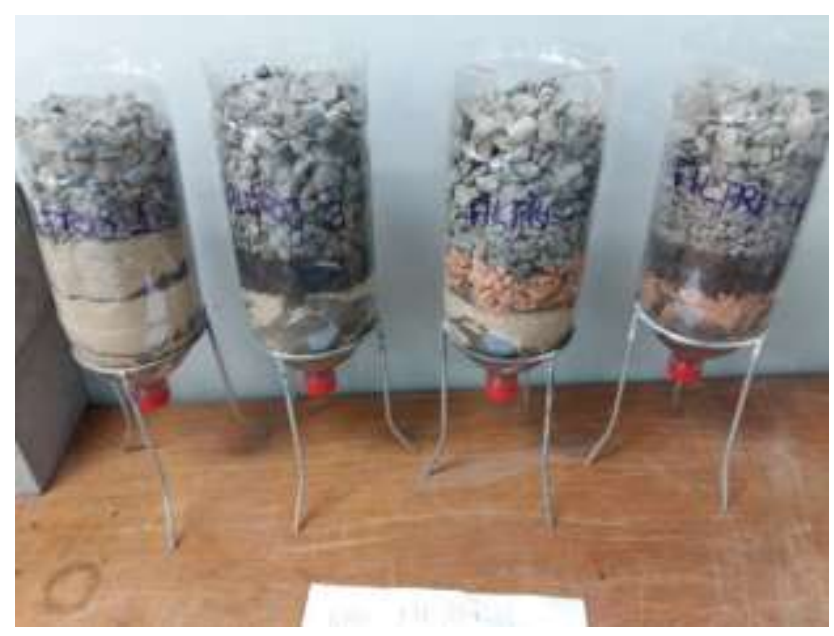

Fonte: Autores (2021).

No filtro 1 foram utilizados os três tipos de areia (fina, média e grossa) e no filtro 2 areia fina e grossa, e após carvão ativado que possui diâmetro de 1-2 mm aumentando a área dos grãos, facilitando a passagem do filtrado. Nas camadas com diâmetros menores, juntamente a areia foi utilizada uma manta geotêxtil para separar as camadas, impossibilitando de se misturarem. Após a montagem dos filtros, foram lavados com água corrente para a limpeza.

\subsection{Análise Físico-Química Do Lixiviado E Determinação Da Vazão}

O lixiviado utilizado para realização dos testes foi coletado da calha Parshall do aterro sanitário de Santa Maria - RS (Figura 2). 
Figura 2. Coleta do lixiviado na Calha Parshall do aterro sanitário.

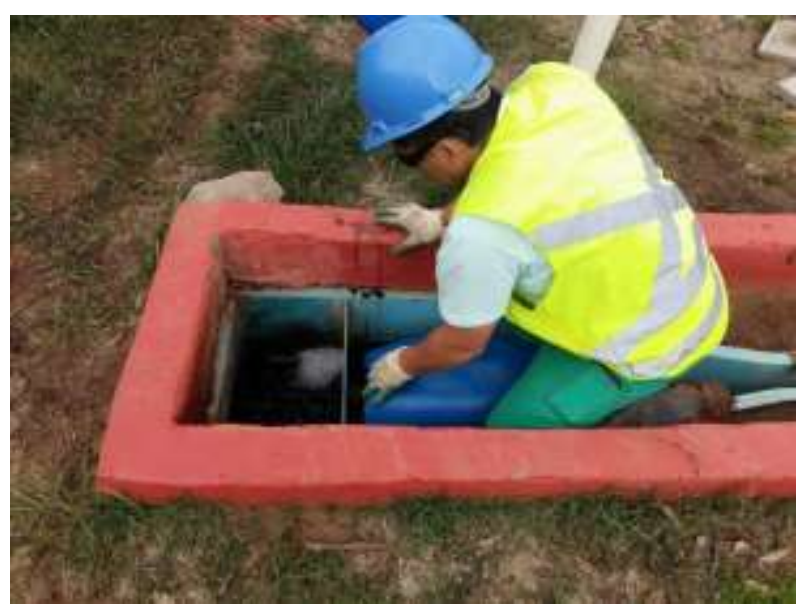

Fonte: Autores (2021).

Os parâmetros físico-químicos que foram analisados no lixiviado bruto, usado como referência, e no filtrado foram: temperatura, potencial hidrogeniônico $(\mathrm{pH})$, oxigênio dissolvido (OD) e condutividade elétrica. Os equipamentos utilizados para determinar os parâmetros foram: termômetro, pHmetro analion PM 608 DIGIMED, oxímetro DM-4 DIGIMED e condutivímetro de bancada digital DM-32 DIGIMED.

Para a determinação da vazão do filtro, foi cronometrado o tempo em que o filtrado preencheu o volume do recipiente. Com as melhores análises do filtrado, foi estimado o volume do filtro em escala maior de vazão para $105 \mathrm{~m}^{3}$.dia-1, analisando se a aplicação do filtro seria considerada viável ou não.

Os dados foram expressos como média \pm desvio padrão (SD). A análise estatística foi realizada por análise de variância (ANOVA) seguida pelo teste de Tukey a $5 \%(\mathrm{P}<0,05)$ quando apropriado.

\section{Resultados e Discussão}

Os parâmetros físico-químicos analisados ( $\mathrm{pH}$, condutividade elétrica, oxigênio dissolvido e temperatura) estão representados na Figura 3. Observa-se que não há diferença significativa no pH dos filtros quando comparado com o lixiviado bruto. A condutividade elétrica mostrou uma redução significativa no filtro 1 ( $\mathrm{p}<0,001$ ), no filtro 2 e 4 (p<0,05) quando comparado com o lixiviado bruto. Ainda, foi observado um aumento significativo no oxigênio dissolvido no filtro 1 e 2 $(\mathrm{p}<0,001)$ e no filtro $4(\mathrm{p}<0,01)$ quando comparado com o lixiviado bruto. Quando foi analisada a temperatura, observou-se uma redução significativa com p<0,001 no filtro 1, 2 e 4 quando comparado com o lixiviado bruto. 
Figura 3. Parâmetros físico-químicos. Analisados por análise da variância (ANOVA) seguida pelo teste de Tukey. Resultados foram expressos como média \pm desvio padrão (SD). *** $\mathrm{p}<0,001, * * \mathrm{p}<0,01 \mathrm{e} * \mathrm{p}<0,05$ quando comparado com o lixiviado bruto.

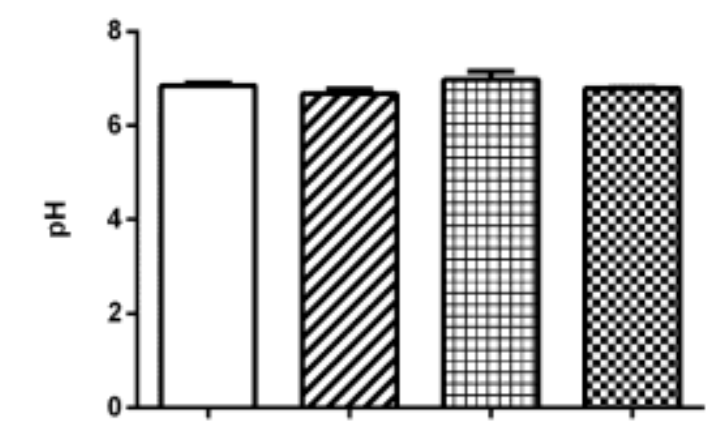

A
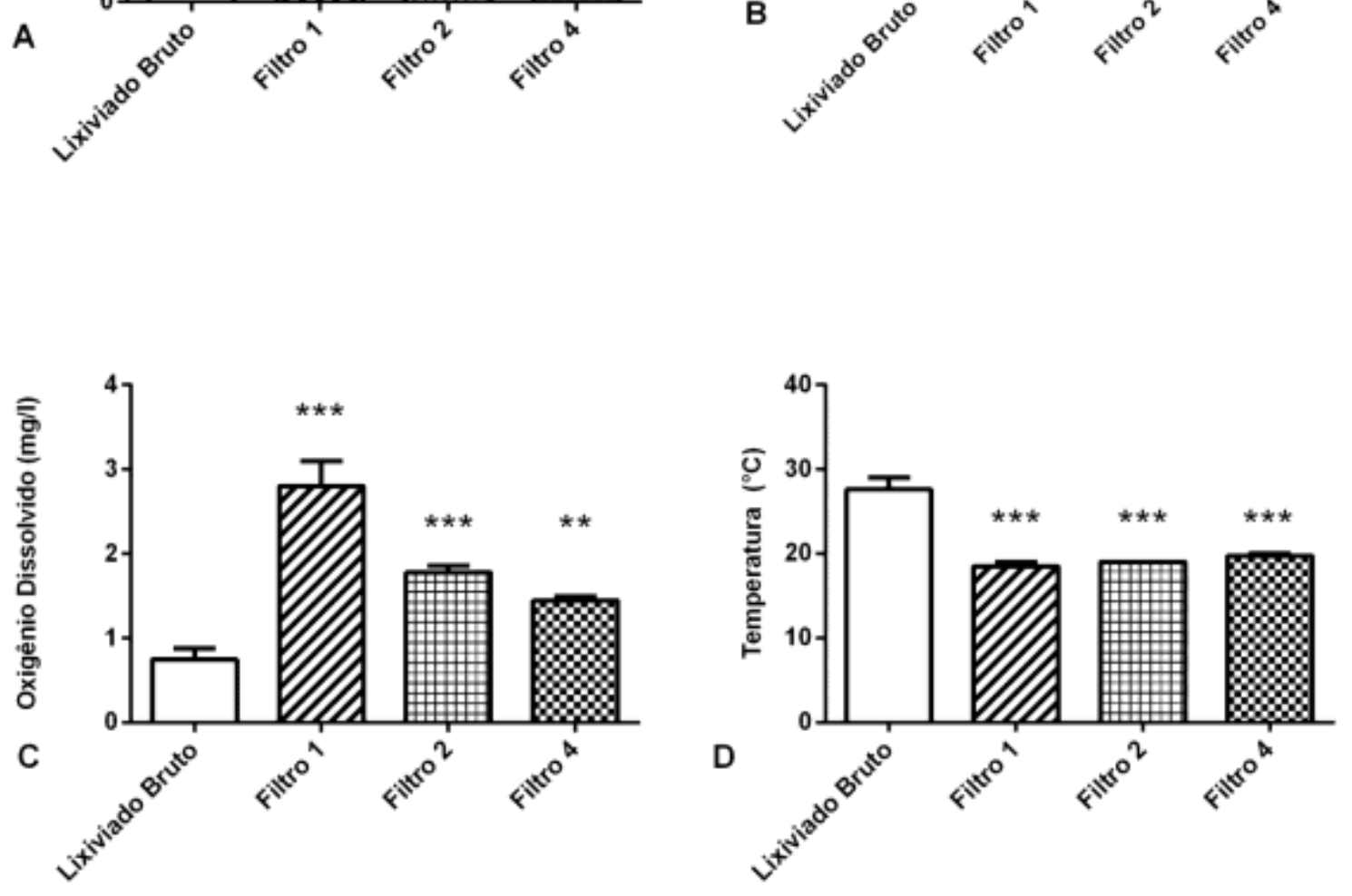

Fonte: Autores (2021).

Na Tabela 2, observa-se a porcentagem dos parâmetros físico-químicos. O pH não reduziu e nem aumentou significativamente nos três tipos de filtro, já a condutividade obteve redução em todos os filtros, mas em maior destaque o filtro 1 , na qual alcançou redução de aproximadamente $50 \%$. O oxigênio dissolvido teve aumento significativo, mas o filtro 1 obteve maior aumento, em torno de $377 \%$. A temperatura reduziu em torno de $30 \%$ nos três tipos de filtro. 
Tabela 2. Porcentagem (\%) da redução dos parâmetros físico-químicos realizados através dos filtros testados.

\begin{tabular}{llcll}
\hline & pH & $\begin{array}{c}\text { Condutividade } \\
\text { elétrica }\end{array}$ & Oxigênio Dissolvido & Temperatura \\
\hline Filtro 1 & 2,34 & 49,87 & 377,75 & 32,96 \\
Filtro 2 & $-1,96$ & 24,36 & 242,78 & 31,05 \\
Filtro 4 & 0,72 & 20,49 & 199,95 & 28,28 \\
\hline
\end{tabular}

Fonte: Autores (2021).

Para garantir o bom funcionamento do sistema de tratamento é necessário que o oxigênio dissolvido seja mantido com os valores acima de $2 \mathrm{mg} . \mathrm{L}^{-1}$, o que se tornou possível depois da passagem do lixiviado pelo filtro. A ausência de oxigênio provoca a morte de todos os demais seres vivos aeróbicos, então com o aumento do oxigênio dissolvido no processo de filtragem ocorre uma melhora na decomposição da matéria orgânica durante o tempo de armazenamento do lixiviado até passar por tratamentos mais avançados, diminuindo sua carga orgânica.

O aumento de $377,75 \%$ do oxigênio dissolvido do filtro 1 mostra sua eficiência na remoção da matéria orgânica do lixiviado bruto. A concentração de matéria orgânica, aliada às altas temperaturas, influencia no grau de desoxigenação da água, devido aos processos de estabilização da matéria orgânica realizados pelas bactérias decompositoras, as quais utilizam o oxigênio disponível no meio líquido para a sua respiração (Silva, 2012; Pantoja et al., 2016). Logo, conclui-se que a matéria orgânica e o oxigênio dissolvido possuem uma relação inversamente proporcional, quanto maior a presença de matéria orgânica no curso d'água, menor será a concentração de OD.

Além disso, pôde-se observar que o OD é um dos parâmetros de qualidade da água mais importantes, pois representa um grande indicativo de poluição hídrica. A quantidade de matéria orgânica no esgoto representa a causa de um dos principais problemas de poluição das águas, em alta concentração causa o aumento de microrganismos e, consequentemente, o consumo excessivo do oxigênio dissolvido nos processos metabólicos de utilização e estabilização da matéria orgânica (Cunha \& ferreira, 2006; Silva, 2012).

A condutividade elétrica representa a quantidade de sais na água. Este parâmetro mostrou uma diminuição significativa após o processo de filtração com areia e brita, já os demais materiais filtrantes como argila e carvão obtiveram menor redução. $\mathrm{O}$ ph se tornou estável após do processo de filtragem.

Na figura 4, está representada a vazão do lixiviado no filtro 1, 2 e 4, pode-se observar diferença significativa entre os filtros ( $\mathrm{p}<0,001)$. O filtro 1 e o 4 tiveram menores índices de vazão que o filtro 2 e o filtro 1 maior vazão que o filtro 4. 
Figura 4. Vazão dos Filtros 1, 2 e 4. Analisados por análise da variância (ANOVA) seguida pelo teste de Tukey. Resultados foram expressos como média \pm desvio padrão (SD). As letras a, b e c representam diferença significativa ( $p<0,001)$ entre os filtros.

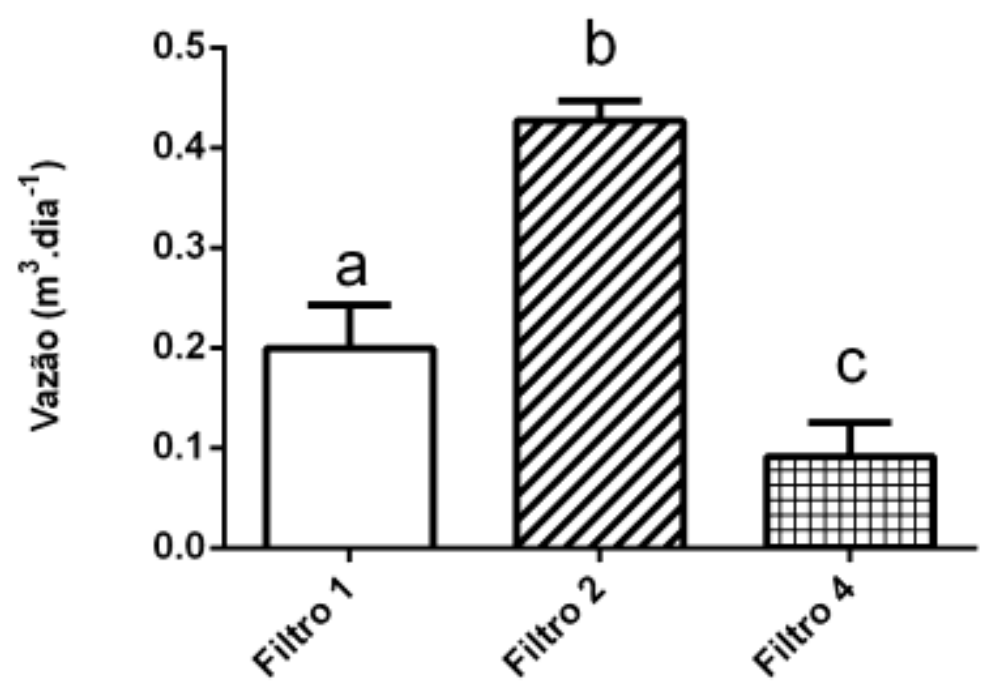

Fonte: Autores (2021).

O filtro 3 não está incluído nas estatísticas porque se tornou irrealizável a filtração, durante a realização dos testes o lixiviado saturou na argila impossibilitando uma vazão viável para ser aplicado em escala maior. Segundo Urach (2007) à medida que aumenta a quantidade dos teores de argila, o solo possui maior capacidade de retenção de água e aumenta a quantidade de água retida no solo. A correlação de retenção de água com argila é explicada pelo efeito da maior adsorção de água em partículas com maior área superficial específica.

A argila também foi usada como material filtrante no filtro 4, por esta razão foi obtida uma menor vazão comparada com o filtro 1 e 2 . Contudo, no filtro 3 havia apenas areia média antes da camada argila, com isto foi possível concluir que uma camada de areia, brita e principalmente, carvão ativado são meios filtrantes necessários de serem usados antes da camada argila, uma vez que eles filtram partículas maiores, reduzindo o tamanho médio das partículas dentro do filtro, aumentando a vida útil da argila como meio filtrante.

Com os resultados descritos, nota-se que o melhor filtro para ser utilizado como pré-tratamento é o filtro 1 , o qual obteve melhor redução dos parâmetros físico-químicos, principalmente redução da condutividade elétrica e uma vazão viável para aplicação do filtro em escala maior, para ser utilizada como pré-tratamento de lixiviado em aterro sanitário.

Então, considerado a vazão do filtro $1 \mathrm{de} 0,2 \mathrm{~m}^{3} \cdot \mathrm{dia}^{-1}$ e a dimensão do filtro redimensionado para uma escala maior sendo de $110 \mathrm{~cm}$ de diâmetro e $150 \mathrm{~cm}$ de altura, este filtro foi considerado como solução viável para sua instalação, pois com isto estima-se que ele possua uma vazão igual ou superior a $105 \mathrm{~m}^{3} \cdot \mathrm{dia}^{-1}$, podendo ser usado como pré-tratamento, antecipando as lagoas de recebimento do lixiviado.

\section{Conclusão}

A partir do deste estudo pode-se concluir que o Filtro 1, composto por areia fina, areia média, areia grossa, brita $\mathrm{n}^{\circ} 0$ e brita ${ }^{\circ} 1$, é o mais indicado para ser utilizado como pré-tratamento do lixiviado, pois, com as análises físico-químicas do filtrado e as vazões determinadas foi o filtro que apresentou os melhores resultados, conseguindo reduzir aproximadamente $50 \%$ da condutividade elétrica e obter $377,75 \%$ de aumento do oxigênio dissolvido. O filtro foi considerado com os melhores 
resultados de viabilidade para ser aplicado em escala maior, considerando uma vazão de $105 \mathrm{~m}^{3} \cdot \mathrm{dia}^{-1}$ para uma dimensão de $110 \mathrm{~cm}$ de diâmetro e $150 \mathrm{~cm}$ de altura, considerado viável a aplicação do mesmo como pré-tratamento antes das lagoas de recebimento de lixiviado.

O presente estudo além de comprovar a eficácia da utilização de filtragem e determinar a composição de filtro ideal para o pré-tratamento do lixiviado do Aterro Sanitário de Santa Maria/RS, cria embasamento para que a técnica seja utilizada em outros aterros, com as devidas adequações, a fim de facilitar os outros tratamentos de lixiviado existentes nas empresas gerenciadoras de aterro sanitário, auxiliando desta forma a atingir os parâmetros físico-químicos adequados perante a legislação vigente.

Contudo, indicamos, para futuras pesquisas, observar outros parâmetros físico-químicos, como por exemplo a Demanda Bioquímica de Oxigênio. Ainda, recomenda-se também avaliar este processo em proporções maiores e com maiores vazões de chorume, pois este estudo foi realizado escala piloto, sendo este um fator restritivo para avaliação do tempo que este material filtrante manterá sua eficiência na remoção dos parâmetros analisados.

\section{Referências}

Araújo, L. G. S. de, Norberto, A. de S., Ferreira, R. P. de S., Mariano, M. O. H., \& Callado, N. H. (2020). Avaliação da eficiência do tratamento de lixiviado de um aterro sanitário. Research, Society and Development, 9(7), e564974466. https://doi.org/10.33448/rsd-v9i7.4466

Associação brasileira de normas técnicas. NBR 8419:1992: Apresentação de projetos de aterros sanitários de resíduos sólidos urbanos. ABNT, 1992. 7p.

Associação brasileira de normas técnicas. NBR 7214:1982: Areia normal para ensaio de cimento - Especificação. ABNT, 1982. 7p.

Bidone, F. R. A., Povinelli, j., \& Cotrim, S. D. S. (1997). Tratamento de lixiviado de aterro sanitário através de filtros percoladores. In $19^{\circ}$ Congresso Brasileiro de Engenharia Sanitária e Ambiental (p. 19). ABES.

Campos, J. C., Moura, D., Costa, A. P., Yokoyama, L., Araujo, F. V. D. F., Cammarota, M. C., \& Cardillo, L. (2013). Evaluation of pH, alkalinity and temperature during air stripping process for ammonia removal from landfill leachate. Journal of Environmental Science and Health, Part A, 48(9), 1105-1113. https://doi.org/10.1080/10934529.2013.774658

Castilhos Junior, A. B. D., Dalsasso, R. L., \& Rohers, F. (2010). Pré-tratamento de lixiviados de aterros sanitários por filtração direta ascendente e coluna de carvão ativado. Engenharia Sanitária e Ambiental, 15, 385-392.

Chen, W., Gu, Z., Ran, G., \& Li, Q. (2021). Application of membrane separation technology in the treatment of leachate in China: A review. Waste Management, 121, 127-140. https://doi.org/10.1016/j.wasman.2020.12.002

Cunha, C. D. L. D. N., \& Ferreira, A. P. (2006). Modelagem matemática para avaliação dos efeitos de despejos orgânicos nas condições sanitárias de águas ambientais. Cadernos de Saúde Pública, 22, 1715-1725. https://doi.org/10.1590/S0102-311X2006000800020

Dia, O., Drogui, P., Buelna, G., \& Dubé, R. (2018). Hybrid process, electrocoagulation-biofiltration for landfill leachate treatment. Waste Management, 75, 391-399. https://doi.org/10.1016/j.wasman.2018.02.016

Dhar-Chowdhury, P., Haque, C. E., \& Driedger, S. M. (2016). Dengue disease risk mental models in the city of Dhaka, Bangladesh: juxtapositions and gaps between the public and experts. Risk Analysis, 36(5), 874-891. https://doi.org/10.1111/risa.12501

Duan, Z., Scheutz, C., \& Kjeldsen, P. (2021). Trace gas emissions from municipal solid waste landfills: A review. Waste Management, 119, 39-62. https://doi.org/10.1016/j.wasman.2020.09.015

Ferri, G. L., Chaves, G. D. L. D., \& Ribeiro, G. M. (2014). Análise e localização de centros de armazenamento e triagem de resíduos sólidos urbanos para a rede de logística reversa: um estudo de caso no município de São Mateus, ES. Production, 25, 27-42. https://doi.org/10.1590/S0103-65132014005000014

Irazusta, S. P., Vieira, C. C., Degasperi, F. T. \& Piacente, F. J. (2020). Determinação da capacidade adsortiva (Ca) de carvão ativado granulado (Cag) usado em filtros químicos para retenção de vapores orgânicos. Research, Society and Development, 9(11), e1809119662. https://doi.org/10.33448/rsd-v9i11.9662

Leite, N. M. G., da Silva Pinheiro, A. R., Madeira, C. S. R., de Brito, R. M., de Souza, M. O. A. J., Araújo, C. H. D. N. L., \& do Nascimento Ribeiro, G. (2021). A influência da disposição final dos resíduos sólidos nos recursos hídricos: uma revisão sistemática. Brazilian Journal of Development, 7(2), 1299713006. https://doi.org/10.34117/bjdv7n2-084

Maler, C. L., Simionato, L. R., Santos, T. A., \& Fernandes, F. (2015). Sequência de tratamentos para lixiviado de aterro sanitário utilizando os processos físico-químico convencional e Fenton como pós-tratamento. Revista DAE, 199, 47-57. https:// doi:10.4322/dae.2014.145

Mucelin, C. A., \& Bellini, M. (2008). Lixo e impactos ambientais perceptíveis no ecossistema urbano. Sociedade \& natureza, $20,111-124$.

Pantoja, N. G. P., Castro, L. M., Rocha, S. D., Silva, J. A., Ribeiro, J. S. P., Donald, A. R., \& Oliveira, T. C. D. S. (2016). Quality of the Solimões River water for domestic use by the riverine community situated in Manacapuru-Amazonas-Brazil. Environmental Science and Pollution Research, 23(12), 11395-11404. https://doi.org/10.1007/s11356-015-5025-2 
Research, Society and Development, v. 10, n. 9, e52110918211, 2021

(CC BY 4.0) | ISSN 2525-3409 | DOI: http://dx.doi.org/10.33448/rsd-v10i9.18211

Queiroz, L. M., Amaral, M. S., Morita, D. M., Yabroudi, S. C., \& Sobrinho, P. A. (2011). Aplicação de processos físico-químicos como alternativa de pré e pós-tratamento de lixiviados de aterros sanitários. Engenharia Sanitária e Ambiental, 16, 403-410. https://doi.org/10.1590/S1413-41522011000400012

Serafim, A. L., Gussakov, K. C., Silva, F., Coneglian, M. R., De Brito, N. N., Sobrinho, G. D., Tonso, S., Pelegrini, R. Chorume, impactos ambientais e possibilidades de tratamentos, in: III Fórum de Estudos Contábeis. Faculdades Integradas Claretianas, Rio Claro, SP, Brasil, 2003.

Silva, L. M. D. (2012). Estudo da correlação entre o oxigênio dissolvido e a matéria orgânica em diferentes ambientes aquáticos da região Amazônica. Dissertação de mestrado. Universidade Federal do Amazonas. Manaus, AM, Brasil. https://tede.ufam.edu.br/bitstream/tede/3341/4/ Luana\%20Monteiro\%20da\%20Silva.pdf

Silva, A. C., Silva, E. M. S., Lima, F. V., \& Santos, M. (2014). Degradação de meios filtrantes na filtragem a vácuo. HOLOS, 3, 309-318. https://doi.org/10.15628/holos.2014.1773

Souza, W. M. de, de Oliveira, I. S., \& Aragão, J. S. (2020). Gestão dos resíduos sólidos em comunidades rurais: um estudo de caso do Sítio Estrela, Barbalha, Estado do Ceará, Brasil. Research, Society and Development, 9(9), e99997057. https://doi.org/10.33448/rsd-v9i9.7057

Urach, F. L. (2007). Estimativa da retenção de água em solos para fins de irrigação. Dissertação de mestrado. Universidade Federal de Santa Maria. Santa Maris, RS, Brasil. Disponível: https://repositorio.ufsm.br/bitstream/handle/1/7593/Binder1.pdf?sequence=1\&isAllowed=y

Vasconcelos, D. V., Silva, L. L. S., Guerra, I. L., \& Campos, J. C. (2017). Estudo da tratabilidade de lixiviado gerado em um aterro controlado. Revista Ambiente \& Água, 12, 457-467. https://doi.org/10.4136/ambi-agua.1957

Zhang, Z., Su, Y., Zhu, J., Shi, J., Huang, H., \& Xie, B. (2021). Distribution and removal characteristics of microplastics in different processes of the leachate treatment system. Waste Management, 120, 240-247. https://doi.org/10.1016/j.wasman.2020.11.025

Wiszniowski, J., Robert, D., Surmacz-Gorska, J., Miksch, K., \& Weber, J. V. (2006). Landfill leachate treatment methods: A review. Environmental chemistry letters, 4(1), 51-61. https://doi.org/10.1007/s10311-005-0016-z

Wu, X., Yue, B., Huang, Q., Wang, Q., Lin, Y., Zhang, W., \& Yan, Z. (2017). Spatio-temporal variation of landfill gas in pilot-scale semi-aerobic and anaerobic landfills over 5 years. Journal of Environmental Sciences, 54, 288-297. https://doi.org/10.1016/j.jes.2016.09.015 